\title{
Multipurpose heterofunctional dendritic scaffolds as crosslinkers towards functional soft hydrogels and implant adhesives in bone fracture applications $\dagger$
}

Cite this: J. Mater. Chem. B, 2013, 1, 6015

Received 30th July 2013

Accepted 27th September 2013

DOI: $10.1039 / \mathrm{c} 3 \mathrm{tb} 21061 \mathrm{~h}$

\author{
Yvonne Hed, ${ }^{a}$ Kim Öberg, ${ }^{a}$ Sandra Berg, ${ }^{a}$ Axel Nordberg, ${ }^{\text {ab }}$ Hans von Holst ${ }^{\text {b }}$ \\ and Michael Malkoch*a
}

www.rsc.org/MaterialsB

\begin{abstract}
Two sets of heterofunctional dendritic frameworks displaying an inversed and exact number of ene and azide groups have successfully been synthesized and post-functionalized with biorelevant molecules. Their facile scaffolding ability enabled the fabrication of soft and azide functional dendritic hydrogels with modulus close to muscle tissue. The dendritic scaffolds are furthermore shown to be promising primers for the development of novel bone fracture stabilization adhesives with shear strengths succeeding commercial Histroacryl ${ }^{\circledR}$.
\end{abstract}

The increasing demand for multi-purpose soft materials for advanced applications has intensified the need for highly controlled synthetic protocols of polymeric materials. ${ }^{1}$ Typically, such materials are of well-defined or even monodisperse nature and display a defined number of several orthogonal functional groups. The construction of these structures relies on the use of efficient, robust and chemoselective reactions. The click concept, as described by Sharpless and coworkers, is today recognized as a set of reactions that fulfill such criteria. ${ }^{2}$ This concept of reliable reactions enables facile access to advanced polymeric structures. ${ }^{3}$ For instance, the combination of controlled polymerization techniques with copper catalyzed azide-alkyne cycloaddition (CuAAC) has resulted in a myriad of sophisticated macromolecules with defined functions. ${ }^{4-6}$ Heterofunctional dendrimers, being synthetically one of the most challenging macromolecular scaffolds to isolate, have also been reported via elegant click strategies. ${ }^{7-12}$

Their globular conformation coupled with the exact number of orthogonal functional groups distributed on the dendritic framework makes these structures promising

\footnotetext{
${ }^{a}$ KTH Royal Institute of Technology, Polymer and Fiber Technology, Division of Coating Technology, Stockholm, Sweden. E-mail: malkoch@kth.se

${ }^{b}$ KTH Royal Institute of Technology, School of Technology and Health, Division of Neuronic Engineering, Stockholm, Sweden

† Electronic supplementary information (ESI) available. See DOI: $10.1039 / \mathrm{c} 3 \mathrm{tb} 21061 \mathrm{~h}$
}

materials for applications such as drug delivery, magnetic resonance imaging and tissue engineering. ${ }^{\mathbf{1 3 , 1 4}}$ Moreover, the copper-free and UV initiated thiol-ene coupling (TEC) reaction, nowadays also included in the click reaction family, ${ }^{15}$ has fueled the field of dendrimer chemistry yielding monodisperse dendrimers via both traditional and accelerated synthetic approaches. ${ }^{16-18}$ In fact, the combination of TEC and CuAAC click reactions enabled the construction of a $6^{\text {th }}$ generation dendrimer in 6 reaction steps exhibiting 192 functional groups and a molecular weight close to $60 \mathrm{kDa} .^{19}$ The modular nature of the UV initiated TEC reaction is recognized as chemoselective to a wide range of functional groups and can proceed under benign reaction conditions in aprotic as well as protic solvents in the presence of oxygen. ${ }^{15}$ The TEC reaction, originally used in coating applications, has most recently been explored for the preparation of hydrogels with controlled modulus and swelling properties ${ }^{\mathbf{2 0 , 2 1}}$ as well as in the presence of biological cells at cytocompatible levels of UV light at 365 $\mathrm{nm}$ and $10 \mathrm{~mW} \mathrm{~cm}{ }^{-2} \cdot{ }^{22}$ Although efficient, most TEC networks reported are neutral and prepared from two component monomer or polymer systems of $\mathrm{A}_{2}$ and $\mathrm{B}_{3}$ with $\mathrm{A}$ and $\mathrm{B}$ being either a thiol or an alkene functionality. However, to deliver crosslinked networks with desired functions the design of components that can undergo crosslinking and in parallel carry additional functions are foreseen as highly attractive probes. ${ }^{23}$ In this report, two sets of orthogonally functionalized and branched scaffolds are prepared displaying a switchable reactive group density of alkenes and primary azides. Their dual-purpose potential was first exploited for the development of a small library of bioactive HFDs via the robust CuAAC reaction. The generated scaffolds were thereafter exploited for the fabrication of soft dendritic hydrogels as well as the use as primers in bone fracture stabilization adhesives.

Heterofunctional dendritic (HFD) scaffolds were sought out as promising monodisperse materials that deliver an exact number orthogonal groups. ${ }^{8}$ The accurate structural build-up with "inverted" bifunctionality capitalized on the synthesis of two distinctively different Trizma ${ }^{\circledR}$-based $\mathrm{AB}_{2} \mathrm{C}$ monomers, 1 

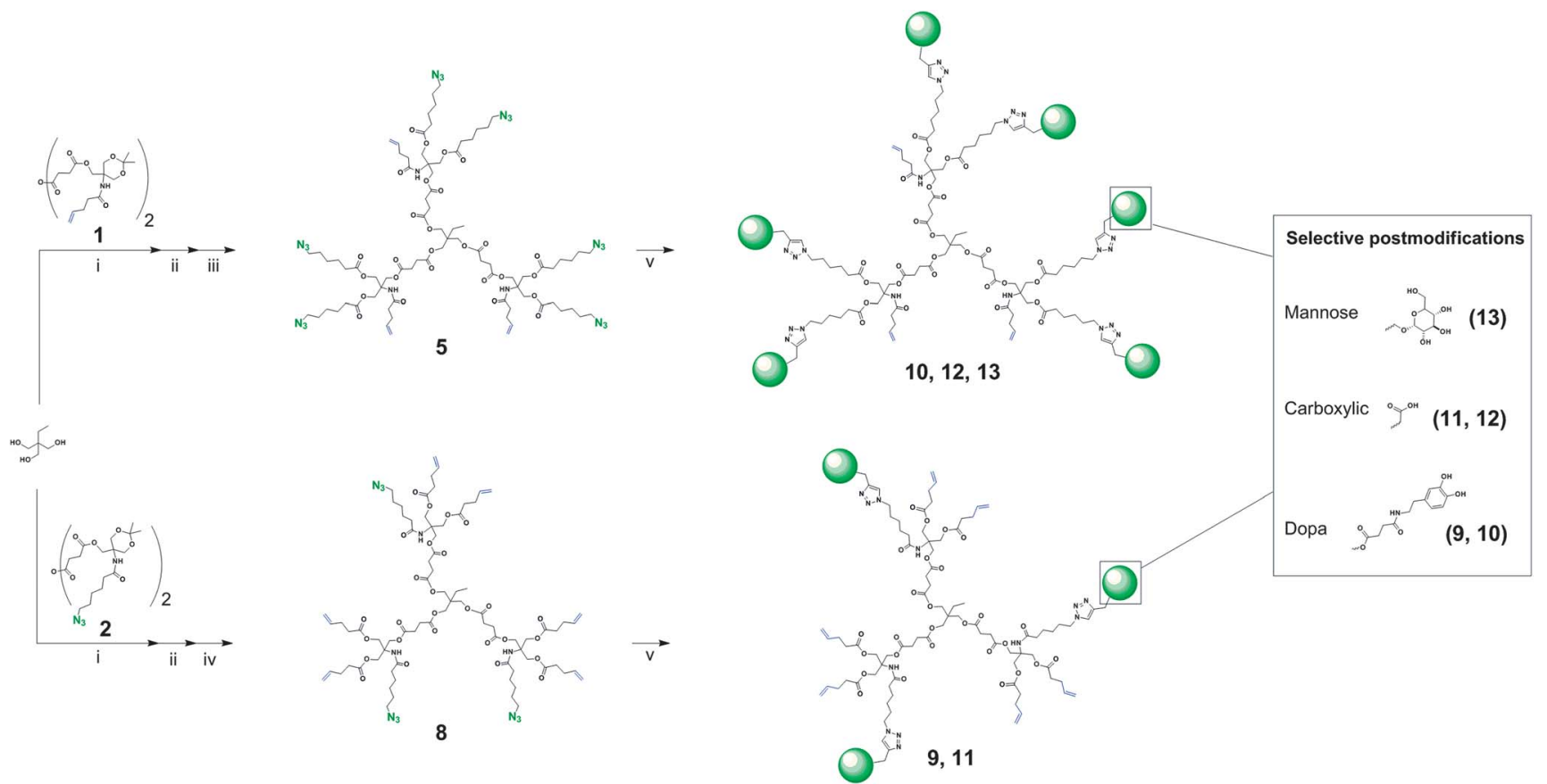

Scheme 1 Synthesis of heterofunctional dendritic scaffolds with interchanging functional group densities of three or six reactive sites. (i) DMAP, pyridine in DCM (ii) 3 or 6, p-TSA in THF- $\mathrm{H}_{2} \mathrm{O}$ (iii) 4, DMAP, pyridine, azide anhydride (18) in DCM (iv) 7, DMAP, pyridine, 4-pentenoic anhydride in DCM (v) acetylene functional (DOPA) (16), 4-pentanoic acid or acetylene functional mannose (21), $\mathrm{NaAsc}_{1} \mathrm{CuSO}_{4}$, in $\mathrm{THF}-\mathrm{H}_{2} \mathrm{O}$.

and 2, displaying both acetonide protected hydroxyls and ene or azide groups (see $\operatorname{ESI} \dagger$ ). As can be seen in Scheme 1, by employing a divergent growth strategy from the trimethylol propane (TMP) core and in combination with $\mathrm{AB}_{2} \mathrm{C}$ monomers, two acetonide protected dendritic scaffolds with three enes 3 $\left(\text { HFD(ei)-G1-e-(Ac) }{ }_{3} \text {-i-(ene }\right)_{3}$ ) or three azides (HFD(ei)-G1-e-(Ac) $)_{3}$ i-(azide) $\left.)_{3}\right) 6$ were efficiently synthesized in gram scales. Subsequent acidic deprotections using pTSA in THF-water solution followed by mild esterification reactions with 6-azidohexanoic anhydride or 4-pentenoic anhydride resulted in scaffold

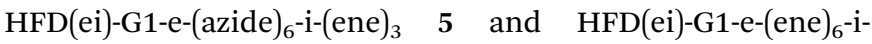
(azide) $)_{3} 8$ exhibiting reactive enes and azides with "inverted" functional group density of three and six. The reactions were monitored by both NMR and MALDI-ToF MS techniques. Upon completion, conventional column chromatography protocols were used, resulting in HFD scaffolds isolated in overall yields of $40 \%$ and $53 \%$, Table 1 .
Satisfied with the purity and dual functionality of these scaffolds, a robust post-functionalization protocol for the introduction of biorelevant groups was adopted. Notably, both azide and ene groups, expressed on these HFD constructs, have separately been used for the fabrication of crosslinked networks. ${ }^{17,25,26}$ In this case, the benign UV initiated TEC chemistry between enes and thiols was identified as a feasible crosslinking strategy whereas the CuAAC click reaction was chosen as a synthetic route for post-functionalization purposes. The multiple azides were selectively reacted with acetylene functional D-mannose, 3,4-dihydroxyphenylalanine (DOPA) and carboxylic acid. All post-functionalization reactions were carried out in THF-water solution and efficiently executed at room temperature in the presence of a catalytic amount of copper sulphate and sodium ascorbate as a copper reducing agent, Table 1. The successful conjugations were supported by MALDIToF and NMR. For example, Fig. 1a shows two isolated

Table 1 Library of heterofunctional dendritic scaffolds

\begin{tabular}{|c|c|c|c|c|}
\hline HFD scaffold & Interior group (3) & Exterior group (6) & $m / z$ calculated $\left[\mathrm{M}+\mathrm{Na}^{+}\right]$ & $m / z$ obtained $\left[\mathrm{M}+\mathrm{Na}^{+}\right]$ \\
\hline 4 & Ene & $\mathrm{OH}$ & 1012.45 & 1012.32 \\
\hline 5 & Ene & Azide & 1846.89 & 1847.03 \\
\hline 7 & Azide & $\mathrm{OH}$ & 1183.55 & 1183.44 \\
\hline 8 & Azide & Ene & 1675.80 & 1675.59 \\
\hline 9 & DOPA & Ene & 2549.13 & 2550.67 \\
\hline 10 & Ene & DOPA & 3593.56 & 3594.67 \\
\hline 11 & $\mathrm{COOH}$ & Ene & 1969.91 & 1970.14 \\
\hline 12 & Ene & $\mathrm{COOH}$ & 2435.12 & 2435.47 \\
\hline 13 & Ene & Mannose & 3156.37 & 3156.86 \\
\hline
\end{tabular}



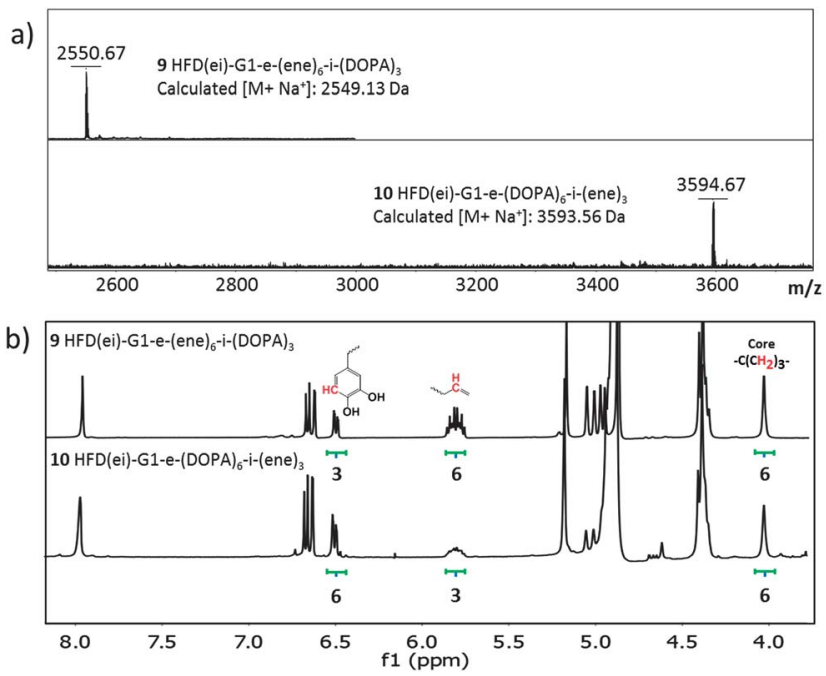

Fig. 1 MALDI-ToF and ${ }^{1} \mathrm{H}-\mathrm{NMR}$ analysis of HFD(ei)-G1-e-(ene) ${ }_{6}-\mathrm{i}$-(DOPA) ${ }_{3} 9$ and HFD(ei)-G1-e-(DOPA) ${ }_{6}-\mathrm{i}$-(ene) ${ }_{3} \mathbf{1 0}$ with inversed functionalities. Nomenclature used as proposed for multifunctional dendrimers where a represent the exterior groups and i the interior groups. ${ }^{24}$

constructs based on the reaction between acetylene functional DOPA and HFD frameworks $\mathbf{5}$ and $\mathbf{8}$. As can be seen by MALDIToF, complete and selective reactions resulted in the formation of HFD(ei)-G1-e-(ene $)_{6}$-i-(DOPA) $)_{3} 9$ and HFD(ei)-G1-e-(DOPA) $)_{6}$-i$(\mathrm{ene})_{3} \mathbf{1 0}$ with inverted bioactive group density and molecular weights corresponding to calculated values. The structural integrity was further corroborated by ${ }^{1} \mathrm{H}-\mathrm{NMR}$, Fig. $1 \mathrm{~b}$.

One driving force to construct these elegant heterofunctional scaffolds was to conceptually assess their modular nature as crosslinkers that allow facile and accurate introduction of additional functionalities within 3D networks. Consequently, their scaffolding ability was evaluated towards the fabrication of soft and functional crosslinked hydrogels as well as novel primers for increased fixation of bone stabilization patches. ${ }^{27}$ For the development of hydrogels, polyethylene glycol (PEG) based networks were targeted as these systems are excellent test beds that today are used as cell culture scaffolds, ${ }^{28}$ targeted drug delivery $^{29}$ and artificial extracellular matrices (aECMs). ${ }^{30}$ Furthermore, their high water content, low immunogenicity and the ability to create well defined structures with tuneable physiochemical properties make these synthetic scaffolds a versatile tool for the design of novel cell culture scaffolds. ${ }^{31}$ However, the inherent inertness toward protein adsorption and lack of cell recognition sites for PEG usually requires the introduction of additional biorelevant chemistries to provide these materials with cell interaction capabilities. One increasingly popular strategy for the manufacturing of PEG hydrogels and simultaneous introduction of such functions has been to use bioorthogonal click chemistries. ${ }^{32}$ In order to demonstrate a simple approach to bioactive hydrogels we herein fabricate azide functional PEG hydrogels utilizing the pre-synthesized dendritic scaffolds as multifunctional dendritic crosslinkers,

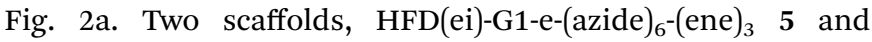
HFD(ei)-G1-e-(ene $)_{6}$-(azide) $)_{3} \mathbf{8}$, were exploited as crosslinkers a)
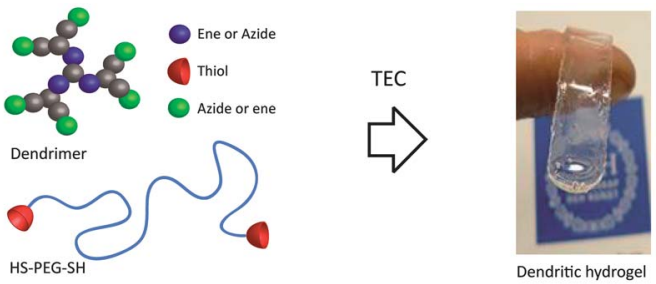

b)

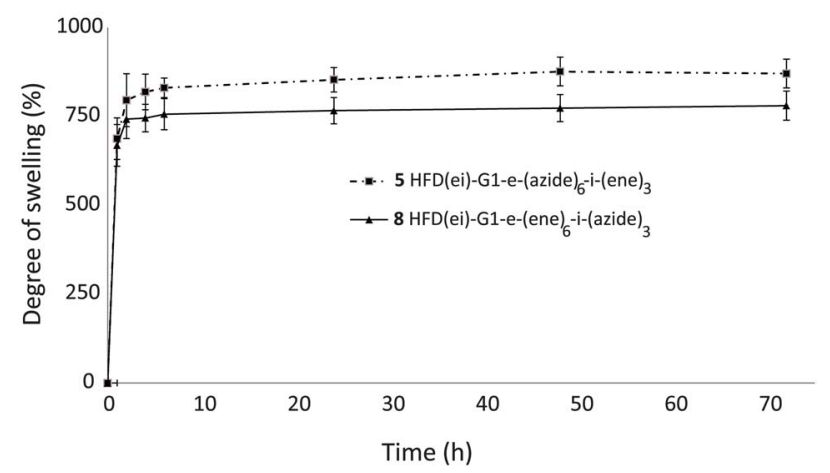

Fig. 2 (a) A schematic representation for the fabrication of a dendritic hydrogel (b) swelling profile of the crosslinked hydrogels.

through a step-growth thiol-ene fashion with a PEG $6 \mathrm{kDa}$ dithiol 14 (see ESI $\dagger$ ). The hydrogels were cured in an ethanol solution within 10 min using $0.5 \mathrm{wt} \%$ of Irgacure 2959 as a photoinitiator $\left(365 \mathrm{~nm}, 9 \mathrm{~mW} \mathrm{~cm}{ }^{-2}\right) .{ }^{22}$ After solvent exchange, from EtOH to water, the water swelling capability was monitored, Fig. 2b. The HFD(ei)-G1-e-(azide) $)_{6}$-(ene $)_{3} 5$ crosslinked hydrogels showed swelling equilibrium at $870 \%$ and a Young's modulus $(E)$ of $21.7 \pm 2.5 \mathrm{kPa}$, which is in close vicinity of muscle tissue (8-17 kPa). ${ }^{33}$ By crosslinking the PEG $6 \mathrm{kDa}$ with the inverted dendritic scaffold, $\operatorname{HFD}(\text { ei)-G1-e-(ene) })_{6}$-(azide) $)_{3} \mathbf{8}$, exposing twice the amount of enes the Young's modulus $(E)$ was more than doubled $(42.8 \pm 2.3 \mathrm{kPa})$. Moreover, this increased functionality of the crosslinker also led to a slight decrease in equilibrium swelling to $780 \%$.

In another context, bone fractures near sensitive tissue, in thin, fragmented or weak bones are of major health care concern since these cannot be optimally repaired using traditional methods including metal screws and plates. The fractured areas are too sensitive for such harsh surgery and therefore development of more sensitive protocols is crucial. Even if metal screws and plates are well accepted as the golden standard in, for instance, iatrogenic bone fractures during neurosurgical and reconstructive procedures, the demand for improved standard technologies is increasing. An alternative approach is to use adhesives for proper fixation of fractured bone. ${ }^{35,36}$ However, applying adhesives within the fracture area itself has been shown to interfere with the natural bone healing process and also sometimes gives rise to insufficient adhesive area. ${ }^{37}$ To minimize the direct use of adhesives on traumatized area, fiber reinforced adhesive patch (FRAP) protocols have been proposed as promising replacements. ${ }^{38}$ This topological procedure benefits from an increased adhesive-to-bone surface area and additionally avoids interference with the natural bone 
healing process within the fracture site. ${ }^{27} \mathrm{~A}$ feasible developed strategy, Fig. 3a, consisted of a two component triazine-based thiol-ene glue system, with six layers of imbedded fibers, that on exposure to UV (320-390 nm, $\left.1.45 \mathrm{~J} \mathrm{~cm}^{-2}\right)$ resulted in crosslinked FRAPs with promising bone stabilizing features. ${ }^{34}$ While such FRAPs were shown to be mechanically strong, their adhesive properties were found poor towards wet bovine femur bones with a shear strength of $0.4 \mathrm{MPa}$, Fig. 3b. This was a consequence of poor interactions between hydrophobic matrices and wet bone surfaces. To increase the interactions, phenolic primers were introduced, for instance poly(parahydroxystyrene), which greatly enhanced the adhesion of FRAPs with a shear strength of $3.4 \mathrm{MPa}^{34}$ In this context, the present heterofunctionality within our dendritic scaffolds was sought as promising primers that can efficiently bind to a wet bone surface as well as undergo covalent crosslinking with a triazine based FRAP system.

As can be seen in Fig. 3b, the patch without any primer resulted in a shear strength of $0.4 \mathrm{MPa}$. The introduction of the dendritic primers at $0.5 \mathrm{wt} \%$ in ethanol increased the shear strength of all FRAPs. While the HFD(ei)-G1-e-(DOPA) ${ }_{6}$-i-(ene) $)_{3}$ 10 primer with the largest number of polar and adhering phenolic groups was initially envisioned to give rise to FRAPs with adhesive properties to wet bone, it was surprisingly found to note the lowest values of all primers with a value of 0.6 MPa. Similar low values, 0.8 MPa, were obtained for HFD(ei)-G1-e$(\mathrm{COOH})_{6}$-i-(ene $)_{3} 12$ with six polar carboxylic groups. These unexpected results are probably due to stronger cohesive forces between the dendritic primers in comparison with the adhesive forces towards the wet bone surfaces. This hypothesis was

a)

b)
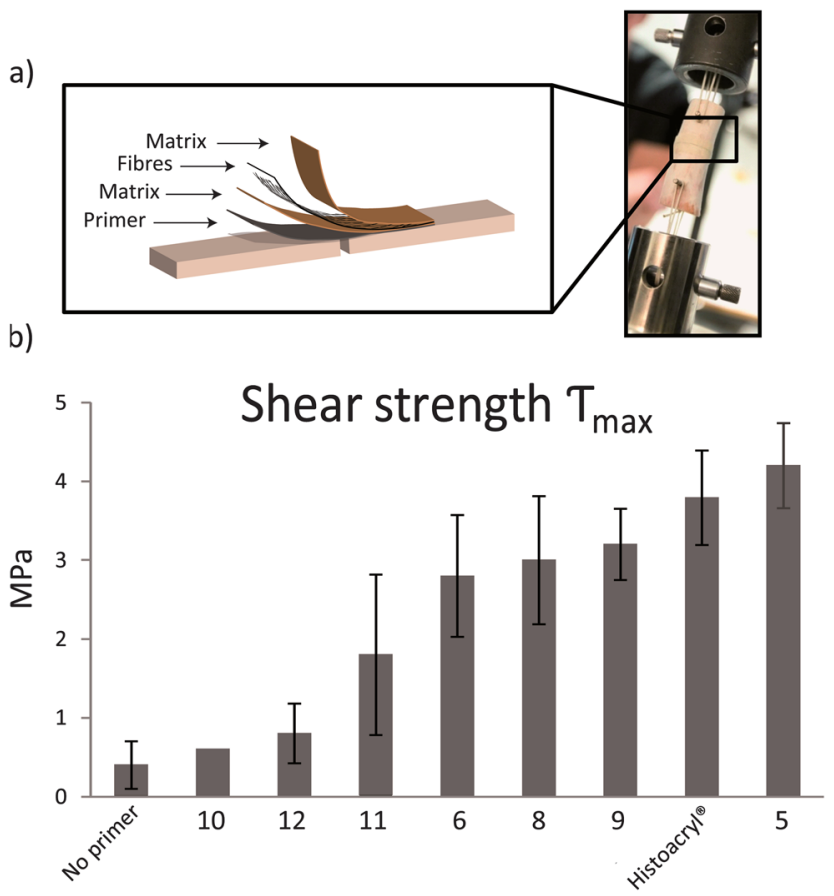

Fig. 3 (a) Illustration of the FRAP on bone (b) maximum shear strengths of the FRAPs using the procedure described previously. ${ }^{34}$ further corroborated by the results obtained for less polar dendritic primers with switched functionalities. In this case, the shear strength of the FRAPs for HFD(ei)-G1-e-(ene $)_{6}$-i-(COOH $)_{3}$ 11 containing three carboxyl groups increased to $1.8 \mathrm{MPa}$ and HFD(ei)-G1-e-(ene) $)_{6}$-i-(DOPA) ${ }_{3} 9$ with three DOPA groups noted a value of 3.2 MPa. None of the dendritic primers decorated with polar groups and enes could deliver FRAPs with adhering strength equivalent to the commercial wound closure adhesive Histoacryl ${ }^{\circledR}$ with an excellent shear strength value of 3.8 MPa. Interestingly, the azide functional $\mathrm{HFD}(\mathrm{ei})-\mathrm{G} 1-\mathrm{e}-(\mathrm{Ac})_{3}$-i-(Azide) ${ }_{3}$ 6 in the absence of enes was found to generate FRAPs with a shear strength value of 2.8 MPa. Further evaluation of HFD(ei)G1-e-(ene) $)_{6}$-i-(Azide) ${ }_{3} 8$ containing an additional six enes resulted in a small increase in adherence to wet bone with a value of 3.0 MPa. These results suggest that the number of available enes plays a minor role while the presence of azides increases the shear strength to a greater extent. Finally, exploiting HFD(ei)-G1-e-(azide) $)_{6}$-(ene) ${ }_{3} \mathbf{5}$ with six azides and three enes as a primer delivered FRAPs with an excellent shear strength of 4.2 MPa that is 10-fold higher than those of the systems without any primers or $10 \%$ higher when compared to Histoacryl ${ }^{3} \cdot{ }^{34} \mathrm{~A}$ potential explanation could be that the azides participate in the crosslinking process during UV exposure of the triazine matrix on the wet substrate. It is already established that alkyl azides rearrange to form reactive imines and nitrogen upon irradiation $^{39}$ and form intermolecular reactions of polymers ${ }^{40}$ as well as participate in reactions leading to intramolecular collapsed dendrimers. ${ }^{8}$ As the imines are intrinsically reactive to themselves, it is here suggested that higher molecular weight primers are generated and efficiently coupled to the bone surface. High molecular weight primers have been previously shown to increase the shear strength of FRAPs on bovine femur bone when compared to low molecular counterparts. ${ }^{34}$ Furthermore, to mimic surgical conditions, the bone surfaces were wet during FRAP fabrication. In the presence of water, the imine will react to produce aldehydes, hydrates and/or acetals. Aldehyde groups are known to react with amino groups in the presence of water, ${ }^{36}$ and therefore may create covalent attachment to the bone surface. One or a combination of these reaction pathways may be the reason for the extraordinary results obtained when using azide functional dendritic materials as primers. It should be noted that both the FRAP matrix and the Trizma ${ }^{\circledR}$ based dendritic scaffolds have previously shown low cytotoxicity towards MG63 osteoblast cells with a cell survival of 95-98\% making this multicomponent FRAP methodology a highly feasible approach towards bone fracture stabilization applications. ${ }^{34}$

In summary, here, we have reported the construction of a library of heterofunctional dendritic scaffolds with clickable azide groups and alkene groups for crosslinking purposes. The careful synthesis of $\mathrm{AB}_{2} \mathrm{C}$-type monomers resulted in monodisperse dendritic frameworks with molecular weights close to $3000 \mathrm{~g} \mathrm{~mol}^{-1}$ with interchanging functional group number within the architectures. Post-functionalization via CuAAC click reaction delivered bioactive materials that were evaluated as dendritic crosslinkers for soft 3D networks as well as primers for the fabrication of fracture stabilization bone adhesives. The 
presence of azide groups within the primers was found to be crucial for the development of highly adhering bone patches with shear strength equivalent to the commercially available Histoacryl ${ }^{\circledR}$. These results highlight the ability of dendritic crosslinkers as adhering components of polymer implants in bone fixation applications on patients suffering from complicated fractures and osteoporosis.

\section{Acknowledgements}

We thank Knut and Alice Wallenberg Foundation (Academy Fellow Grant 2012-0196) and the Swedish Research Council (VR) Grant 2011-5358 and 2010-453.

\section{Notes and references}

1 R. K. Iha, K. L. Wooley, A. M. Nystrom, D. J. Burke, M. J. Kade and C. J. Hawker, Chem. Rev., 2009, 109, 5620-5686.

2 H. C. Kolb, M. G. Finn and K. B. Sharpless, Angew. Chem., Int. Ed., 2001, 40, 2004-2021.

3 P. Lundberg, C. J. Hawker, A. Hult and M. Malkoch, Macromol. Rapid Commun., 2008, 29, 998-1015.

4 P. L. Golas and K. Matyjaszewski, Chem. Soc. Rev., 2010, 39, 1338-1354.

5 K. Kempe, A. Krieg, C. R. Becer and U. S. Schubert, Chem. Soc. Rev., 2012, 41, 176-191.

6 R. K. Iha, K. L. Wooley, A. M. Nystrom, D. J. Burke, M. J. Kade and C. J. Hawker, Chem. Rev., 2009, 109, 5620-5686.

7 P. Wu, M. Malkoch, J. N. Hunt, R. Vestberg, E. Kaltgrad, M. G. Finn, V. V. Fokin, K. B. Sharpless and C. J. Hawker, Chem. Commun., 2005, 5775-5777.

8 P. Antoni, Y. Hed, A. Nordberg, D. Nystrom, H. H. von, A. Hult and M. Malkoch, Angew. Chem., Int. Ed., 2009, 48, 2126-2130.

9 M. I. Montanez, Y. Hed, S. Utsel, J. Ropponen, E. Malmstroem, L. Wagberg, A. Hult and M. Malkoch, Biomacromolecules, 2011, 12, 2114-2125.

10 T. Kang, R. J. Amir, A. Khan, K. Ohshimizu, J. N. Hunt, K. Sivanandan, M. I. Montanez, M. Malkoch, M. Ueda and C. J. Hawker, Chem. Commun., 2010, 46, 1556-1558.

11 R. J. Amir, L. Albertazzi, J. Willis, A. Khan, T. Kang and C. J. Hawker, Angew. Chem., Int. Ed., 2011, 50, 3425-3429.

12 L. Albertazzi, F. M. Mickler, G. M. Pavan, F. Salomone, G. Bardi, M. Panniello, E. Amir, T. Kang, K. L. Killops, C. Brauchle, R. J. Amir and C. J. Hawker, Biomacromolecules, 2012, 13, 4089-4097.

13 M. A. Mintzer and M. W. Grinstaff, Chem. Soc. Rev., 2011, 40, 173-190.

14 C. C. Lee, J. A. MacKay, J. M. J. Frechet and F. C. Szoka, Nat. Biotechnol., 2005, 23, 1517-1526.

15 C. E. Hoyle, A. B. Lowe and C. N. Bowman, Chem. Soc. Rev., 2010, 39, 1355-1387.

16 K. L. Killops, L. M. Campos and C. J. Hawker, J. Am. Chem. Soc., 2008, 130, 5062-5064.
17 M. I. Montanez, L. M. Campos, P. Antoni, Y. Hed, M. V. Walter, B. T. Krull, A. Khan, A. Hult, C. J. Hawker and M. Malkoch, Macromolecules, 2010, 43, 6004-6013.

18 M. V. Walter, P. Lundberg, A. Hult and M. Malkoch, J. Polym. Sci., Part A: Polym. Chem., 2011, 49, 2990-2995.

19 P. Antoni, M. J. Robb, L. Campos, M. Montanez, A. Hult, E. Malmstrom, M. Malkoch and C. J. Hawker, Macromolecules, 2010, 43, 6625-6631.

20 T. Yang, M. Malkoch and A. Hult, J. Polym. Sci., Part A: Polym. Chem., 2012, 51, 363-371.

21 T. Yang, H. Long, M. Malkoch, G. E. Kristofer, L. Berglund and A. Hult, J. Polym. Sci., Part A: Polym. Chem., 2011, 49, 4044-4054.

22 S. J. Bryant, C. R. Nuttelman and K. S. Anseth, J. Biomater. Sci., Polym. Ed., 2000, 11, 439-457.

23 K. Oberg, Y. Hed, R. I. Joelsson, J. Kelly, P. Lowenhielm and M. Malkoch, Chem. Commun., 2013, 6938-6940.

24 M. V. Walter and M. Malkoch, Chem. Soc. Rev., 2012, 41, 4593-4609.

25 M. Malkoch, R. Vestberg, N. Gupta, L. Mespouille, P. Dubois, A. F. Mason, J. L. Hedrick, Q. Liao, C. W. Frank, K. Kingsbury and C. J. Hawker, Chem. Commun., 2006, 2774-2776.

26 C. Nilsson, E. Malmstroem, M. Johansson and S. M. Trey, J. Polym. Sci., Part A: Polym. Chem., 2009, 47, 589-601.

27 A. Nordberg, H. H. von, K. Brolin and A. Beckman, Bio-Med. Mater. Eng., 2007, 17, 299-308.

28 A. M. Kloxin, C. J. Kloxin, C. N. Bowman and K. S. Anseth, Adv. Mater., 2010, 22, 3484-3494.

29 B. Jeong, Y. H. Bae and S. W. Kim, J. Controlled Release, 2000, 63, 155-163.

30 N. Chen, Z. Zhang, B. Soontornworajit, J. Zhou and Y. Wang, Biomaterials, 2012, 33, 1353-1362.

31 N. A. Peppas, J. Z. Hilt, A. Khademhosseini and R. Langer, Adv. Mater., 2006, 18, 1345-1360.

32 M. A. Azagarsamy and K. S. Anseth, ACS Macro Lett., 2013, 2219-2224.

33 A. J. Engler, S. Sen, H. L. Sweeney and D. E. Discher, Cell, 2006, 126, 677-689.

34 A. Nordberg, P. Antoni, M. I. Montanez, A. Hult, H. H. Von and M. Malkoch, ACS Appl. Mater. Interfaces, 2010, 2, 654657.

35 M. Donkerwolcke, F. Burny and D. Muster, Biomaterials, 1998, 19, 1461-1466.

36 B. Hoffmann, E. Volkmer, A. Kokott, P. Augat, M. Ohnmacht, N. Sedlmayr, M. Schieker, L. Claes, W. Mutschler and G. Ziegler, J. Mater. Sci.: Mater. Med., 2009, 20, 2001-2009.

37 S. C. Woodward, Ann. N. Y. Acad. Sci., 1968, 146, 225-250.

38 S. L. Y. Woo, W. H. Akeson, B. Levenetz, R. D. Coutts, J. V. Matthews and D. Amiel, J. Biomed. Mater. Res., 1974, 8, 321-338.

39 Organic Azides Syntheses And Applications, ed. S. Braese and K. Banert, John Wiley \& Sons Ltd., 2010.

40 P. Ling and C. A. Wight, J. Phys. Chem. B, 1997, 101, 21262131. 